\title{
Modulation of Terahertz Polarization on Picosecond Timescales using Polymer-Encapsulated Semiconductor Nanowires
}

\author{
Sarwat A. Baig' ${ }^{1}$, Jessica. L. Boland ${ }^{2}$, Djamshid A. Damry², H. Hoe Tan ${ }^{3}$, Chennupati Jagadish ${ }^{3}$, Hannah J. \\ Joyce $^{1}$, and Michael B. Johnston ${ }^{2}$ \\ ${ }^{I}$ Department of Engineering, University of Cambridge, UK \\ ${ }^{2}$ Department of Physics, Clarendon Laboratory, University of Oxford, UK \\ ${ }^{3}$ Department of Electronic Materials Engineering, Australian National University, Australia
}

\begin{abstract}
We exploit the photoconductivity of semiconductor nanowires to achieve ultrafast broad-bandwidth modulation of $\mathrm{THz}$ pulses. A modulation depth of $-8 \mathrm{~dB}$ was exhibited by a polarizer consisting of 14 layers of nanowires encapsulated in polymer.

OCIS codes: (130.5990) Semiconductors; (160.4236) Nanomaterials; (230.5440) Polarization-selective devices; (250.4110); (300.6495) Spectroscopy, terahertz; Modulators; (310.6628) Subwavelength structures, nanostructures; (320.5390) Picosecond phenomena
\end{abstract}

\section{Introduction}

Enormous advances in terahertz (THz) emitters and detectors have enabled the development of $\mathrm{THz}$ technology in a wide variety of applications, ranging from non-destructive materials evaluation to security screening and medical imaging. THz communications, on the other hand, is in its infancy despite its clear promise for high speed wireless communication. The chief bottleneck facing $\mathrm{THz}$ communications is a lack of practical $\mathrm{THz}$ modulators required to encode information on the $\mathrm{THz}$ waveform.

In this paper we demonstrate $\mathrm{THz}$ polarization modulators with high modulation depth, broad bandwidth and fast optical switching. These THz polarizers are based on GaAs nanowires arranged in a wire-grid configuration that are rendered conductive by photoexcitation. The design mimics conventional wire-grid polarizers constructed from metallic nanowires, but exploits the semiconducting nature of the nanowires with conduction that can be switched "on" via photoexcitation (Fig. 1). When photoexcited by an ultrashort optical pulse, nanowires selectively absorb the component of the incident $\mathrm{THz}$ pulse $(0.1$ to $4 \mathrm{THz})$ polarized parallel to the nanowire axes.

Fig. 1. Schematic diagram illustrating the operation of the THz polarization modulator. Without photoexcitation, the THz pulse is transmitted through the polarizer with minimal absorption (Transmission [1]). Photoexcitation renders the nanowires conductive to absorb the component of the $\mathrm{THz}$ pulse oriented parallel to the nanowire axis (Absorption [0]).

GaAs nanowires are ideal candidates for ultrafast optically switched $\mathrm{THz}$ polarizers as they exhibit (i) large broadband photoconductivity in response to photoexcitation by virtue of their high optical absorption coefficients and high electron mobilities $\left(>1000 \mathrm{~cm}^{2} \mathrm{~V}^{-1} \mathrm{~s}^{-1}\right)$ [1], (ii) rapid $(<5 \mathrm{fs})$ photoconductivity decays due to their short charge carrier lifetimes and high surface recombination velocity [1] and (iii) polarization-dependent absorption of both optical radiation and $\mathrm{THz}$ radiation due to the quasi one-dimensional nanowire geometry [2]. The last property is similar to that of carbon nanotubes, with which nanostructured polarizers have been demonstrated previously (e.g. $[3,4])$. 


\section{Results and discussion}

We employ optical pump-THz probe spectroscopy and vary the polarization of the optical pump beam to selectively photoexcite nanowires oriented parallel to the pump polarization. The polarizers were constructed by embedding the aligned nanowires in a flexible membrane of parylene $\mathrm{C}$ polymer. Films of nanowires-in-polymer were then laminated together to create multilayer films of up to 14 layers. Fig. 2 plots the THz transmission through the polarizers as a function of polarization of the photoexcitation pulse $\left(2 \theta=0^{\circ}\right.$ is polarized parallel to the nanowires and $2 \theta=90^{\circ}$ is polarized perpendicular to the nanowires). Fits to the data show that $\mathrm{THz}$ transmission follows a cosine-squared relationship as expected from Malus's law.

The areal density of nanowires increases with the number of layers of nanowires-in-polymer laminated together. Increasing the nanowire density in turn increases the modulation depth and extinction coefficient of the polarizer. The best performance was observed for the 14-layer polarizer, which exhibited a modulation depth of $8 \mathrm{~dB}$. This is significantly better than ultrafast polarizers based on carbon nanotubes, demonstrated previously [4].

Fig. 2. THz transmission through nanowire polarizers as a function of angle of polarization of the photoexcitation pulse.

After photoexcitation, the photoconductivity of the nanowires decayed rapidly with a single exponential time constant of approximately 1 ps. The transient $\mathrm{THz}$ absorption similarly decays on a picosecond time scale, and this property allows ultrafast modulation of the $\mathrm{THz}$ transmission.

\section{Conclusion}

The nanowire-grid polarizer combines a large modulation depth, broad bandwidth and picosecond time resolution for $\mathrm{THz}$ wave modulation. These properties make it ideal as a modulator in future high-speed, picosecond $\mathrm{THz}$ communication.

\section{References}

[1] H. J. Joyce, C. J Docherty, Q. Gao, H. H. Tan, C. Jagadish, J. Lloyd-Hughes, L. M. Herz and M. B. Johnston, "Electronic properties of GaAs, InAs and InP nanowires studied by terahertz spectroscopy," Nanotechnology, 24, 214006 (2013)

[2] H. J. Joyce, J. L. Boland, C. L. Davies, S. A. Baig, M. B. Johnston, "A review of the electrical properties of semiconductor nanowires: insights gained from terahertz conductivity spectroscopy," Semicond. Sci. Technol., 31, 103003 (2016).

[3] L. Ren, C. L. Pint, L. G. Booshehri, W. D. Rice, X. Wang, D. J. Hilton, K. Takeya, I. Kawayama, M. Tonouchi, R. H. Hauge and J. Kono, "Carbon Nanotube Terahertz Polarizer," Nano Lett, 9, 2610-2613 (2009)

[4] C. J. Docherty, S. D. Stranks, S. N. Habisreutinger, H. J. Joyce, L. M. Herz, R. J. Nicholas, and M. B. Johnston, "An ultrafast carbon nanotube terahertz polarisation modulator," J. Appl. Phys., 115, 203108 (2014). 